\title{
Determining weight-bearing tissue condition using peak reactive hyperemia response trend and ultrasonographic features: implications for pressure ulcer prevention
}

\begin{abstract}
Frequent repositioning is important to prevent pressure ulcer (PU) development, by relieving pressure and recovering damages on skin areas induced by repetitive loading. Although repositioning is the gold standard to prevent PU, there is currently no strategy for determining tissue condition under preventive approaches. In this study, the peak reactive hyperemia (RH) trends and ultrasonographic (US) features are compared with the tissue condition under histopathological examination to determine the potential use of these features in determining the tissue condition noninvasively. Twenty-one male Sprague-Dawley rats (seven per group), with body weight of 385-485 g, were categorized into three groups and subjected to different recovery times, each with three repetitive loading cycles at skin tissues above of right trochanter area. The first, second, and third groups were subjected to short (3 minutes), moderate (10 minutes), and prolonged (40 minutes) recovery, respectively, while applying fixed loading time and pressure (10 minutes and $50 \mathrm{mmHg}$, respectively), to provide different degree of recovery and tissue conditions (tissue damage and tissue recovery). Peak RH was measured in the three cycles to determine RH trend (increasing, decreasing, and inconsistent). All rat tissues were evaluated using ultrasound at pre- and post-experiment and rated by two raters to categorize the severity of tissue changes (no, mild, moderate, and severe). The tissue condition was also evaluated using histopathological examination to distinguish between normal and abnormal tissues. Most of the samples with increasing RH trend is related to abnormal tissue (71\%); while inconsistent RH trends is more related to normal tissue (82\%). There is no relationship between the tissue conditions evaluated under ultrasonographic and histopathological examination. Peak RH trend over repetitive loading may serve as a new feature for determining the tissue condition that leading to pressure ulcer.
\end{abstract}

